\title{
AVALIAÇÃO DA TRAJETÓRIA DOS PARAFUSOS BICORTICAIS PELA TÉCNICA DE HARMS E MELCHER EM RELAÇÃO À ARTÉRIA CARÓTIDA INTERNA: ESTUDO EXPERIMENTAL EM CADÁVERES
}

\author{
EVALUATION OF THE TRAJECTORY OF THE BICORTICAL SCREWS IN RELATION TO THE \\ INTERNAL CAROTID ARTERY, IN FIXATION BY THE HARMS AND MELCHER TECHNIQUE: \\ AN EXPERIMENTAL STUDY IN CADAVERS
}

\author{
EVALUACIÓN DE LA TRAYECTORIA DE LOS TORNILLOS BICORTICALES EN LA TÉCNICA DE \\ HARMS Y MELCHER EN RELACIÓN CON LA ARTERIA CARÓTIDA INTERNA: UN ESTUDIO \\ EXPERIMENTAL EN CADÁVERES
}

Guilherme Zanini Rocha ${ }^{1}$, Aluízio Augusto Arantes Junior ${ }^{2}$, Cassius Vinicius Correa dos Reis ${ }^{3}$, Sebastiāo Nataniel Silva Gusmä0 ${ }^{4}$, Warley Martins ${ }^{5}$, Arthur Adolfo Nicolato ${ }^{5}$, Guilherme Lourenço. Lima. Reis ${ }^{6}$, Fernando Flavio Vasconcelos Gonçalves, , Julio César Boynard Santiago ${ }^{7}$

\begin{abstract}
RESUMO
Objetivo: O objetivo deste trabalho é estudar, em peças anatômicas; a relação entre os parafusos bicorticais pela técnica de Harms e Melcher e a artéria carótida interna. Métodos: Nossa amostra consiste em cinco cadáveres. Resultados: Os resultados encontrados foram: a média da menor distância entre o orifício de saída do parafuso e a borda medial da artéria carótida interna direita foi de 11,55 mm (com variação de 10,05 a 14,23 mm), enquanto do lado esquerdo a média foi de 7,50 mm (variando de 2,75 a 12,42 mm). A média da menor distância entre a borda posterior da artéria carótida interna e a cortical anterior da massa lateral de C1 à direita foi de 4,24 mm (variando de 2,08 a 7,48 mm), enquanto do lado esquerdo a média obtida foi de 2,98 mm (com variação de 1,83 a 3,83 mm). Conclusão: Os resultados encontrados estão de acordo com os estudos similares existentes na literatura que enfatizam a necessidade de uma avaliação imaginológica criteriosa da posição anatômica da artéria carótida interna antes da utilização de parafusos bicorticais na massa lateral de C1 por via posterior.
\end{abstract}

Descritores: Coluna vertebral; Atlas cervical; Parafusos ósseos; Artéria carótida interna; Cadáver; Tomografia.

\begin{abstract}
Objective: The objective of this study is to study the relationship between bicortical screws and the internal carotid artery, in anatomical body parts, in screw fixation by the Harms and Melcher technique. Methods: Our sample consisted of five cadavers. Results: The results were as follows: the average shortest distance between the outlet of the screw and the medial edge of the right internal carotid artery was 11.55 $\mathrm{mm}$ (range 10.05 to $14.23 \mathrm{~mm}$ ), while on the left side, the average was $7.50 \mathrm{~mm}$ (ranging from 2.75 to $12.42 \mathrm{~mm}$ ). The average shortest distance between the posterior edge of the internal carotid artery and the anterior cortical C1 lateral mass on the right was $4.24 \mathrm{~mm}$ (ranging from 2.08 to $7.48 \mathrm{~mm}$ ), while the left side, the average was $2.98 \mathrm{~mm}$ (ranging from 1.83 to $3.83 \mathrm{~mm}$ ). Conclusion: The results are consistent with similar studies in the literature that emphasize the need for a careful assessment of images of anatomical position of the internal carotid artery prior to the use of bicortical screws in the C1 lateral mass by posterior access.
\end{abstract}

Keywords: Spine; Cervical atlas; Bone screws; Carotid artery, internal; Cadaver; Tomography.

\section{RESUMEN}

Objetivo: O objetivo de este estudio es, en las piezas anatómicas, la relación entre los tornillos bicorticales, mediante la técnica de Harms y Melcher, y la arteria carótida interna. Métodos: La muestra se compone de cinco cadáveres. Resultados: Los resultados fueron: la distancia más corta promedio, entre la salida del tornillo y el borde medial de la arteria carótida interna derecha, fue 11,55 mm (rango de 10,05 a 14,23 $\mathrm{mm}$ ), mientras que, en la izquierda, el promedio fue 7,50 mm (rango 2,75 a 12,42 mm). La distancia más corta promedio, entre el borde posterior de la arteria carótida interna y la cortical anterior C1 de la derecha, fue 4,24 mm (que van desde 2,08 hasta 7,48 mm), mientras que, en el lado izquierdo, el promedio fue 2,98 mm. (que van desde 1,83 hasta 3,83 mm). Conclusión: Los resultados son consistentes con estudios similares en la literatura que hacen hincapié en la necesidad de una evaluación cuidadosa de las imágenes y posición anatómica de la arteria carótida interna, antes del uso de tornillos bicorticales de masa lateral de C1 por acceso posterior.

Descriptores: Columna vertebral; Atlas cervical; Tornillos óseos; Arteria carótida interna; Cadáver; Tomografía.

\footnotetext{
1. Ortopedista, pesquisador do Laboratório de Anatomia Neurocirúrgica da Faculdade de Medicina da Universidade Federal de Minas Gerais (UFMG) - Minas Gerais - Brasil.

2. Neurocirurgião, Professor Substituto do Departamento de Cirurgia da Faculdade de Medicina da Universidade Federal de Minas Gerais (UFMG) - Minas Gerais - Brasil.

3. Neurocirurgião, Coordenador do Laboratório de Anatomia Neurocirúrgica da Faculdade de Medicina da Universidade Federal de Minas Gerais ( UFMG) - Minas Gerais - Brasil.

4. Neurocirurgião, Professor Titular do Departamento de Cirurgia da Faculdade de Medicina da Universidade Federal de Minas Gerais (UFMG) - Minas Gerais - Brasil.

5. Acadêmico da Faculdade de Medicina da Universidade Federal de Minas Gerais (UFMG) - Minas Gerais - Brasil.

6. Radiologista, membro do corpo clinico do Hospital MadreTeresa de Belo Horizonte - Minas Gerais - Brasil.

7. Ortopedista, membro do corpo clinico do Hospital Maria Amélia Lins - FHEMIG.
}

Trabalho realizado no Laboratório de Anatomia Neurocirúrgica da Faculdade de Medicina da Universidade Federal de Minas Gerais - MG - Brasil.

Endereço para Correspondência: Faculdade de Medicina - Av. Alfredo Balena, 190 - Sala 127 - CEP: 30130-100 - Belo Horizonte - MG - Brasi - E-mail: guizanini@ yahoo.com.br 


\section{INTRODUÇÃO}

Na ultima década, a literatura tem mostrado uma evolução nas formas de instrumentação para o tratamento das lesões da coluna cervical alta $^{1-4}$, especificamente nas doenças que acometem a articulação atlantoaxial $\left.\right|^{5,6}$

Em 1939, Gallie descreveu a técnica de amarrilho C1-C2, apresentando uma taxa de $25 \%$ de pseudoartrose ${ }^{7}$. Outros autores descreveram suas técnicas, como; Brooks-Jenkins e Sonntag, com taxas de fusão óssea de $93 \%{ }^{8}$ e $97 \%{ }^{9}$, respectivamente. È comum às técnicas de amarrilho; o baixo controle rotacional da articulação atlantoaxial, a necessidade de utilização de imobilização externa por período prolongado, e a obrigatoriedade da integridade dos elementos posteriores de C1 e C2.

Com a introdução dos parafusos, passou a ser possível obter maior controle das forças rotacionais da articulação atlantoaxial e consequentemente, melhores taxas de fusão óssea, sem a necessidade de imobilização externa. Este avanço biomecânico veio acompanhado de maiores taxas de complicações neuro vasculares; sendo a mais enfatizada a lesão da artéria vertebral, presente em $4,1 \%$ a $8 \%$ dos casos, mau posicionamento de parafusos em $14 \%$, paresia do nervo hipoglosso em $2 \%{ }^{10,11}$.

Em 2003 Currier et al. ${ }^{12}$ relataram um caso de mau posicionamento do parafuso transarticular C1-C2 em relação à artéria carótida interna, a partir desse relato, inédito na literatura, poucos estudos foram desenvolvidos abordando este tema.

As vias de acesso anterior ${ }^{13}$ e posterior ${ }^{14}$ podem ser utilizadas, sendo a ultima mais familiar ao cirurgião de coluna. As complicações vasculares associadas aos modernos métodos de fixação posterior antlantoaxial incluem não só o risco de lesão das artérias vertebrais $^{15}$, como também das artérias carótidas internas, lesões potencialmente graves e ainda pouco estudadas ${ }^{16}$

Entre as técnicas de fixação posterior de C1-C2 destacam-se as descritas por Harms e Melcher ${ }^{5}$ e Margerl e Seeman ${ }^{6}$, bastante utilizadas, cada qual com as suas limitações ${ }^{15,17}$. Uma característica em comum das técnicas citadas é a utilização de parafuso bi cortical para estabilização da massa lateral do Atlas (C1). Diversos estudos comprovam a superioridade biomecânica dos parafusos bi corticais sobre os parafusos uni corticais ${ }^{18-20}$ ( Figuras 1 e 2).

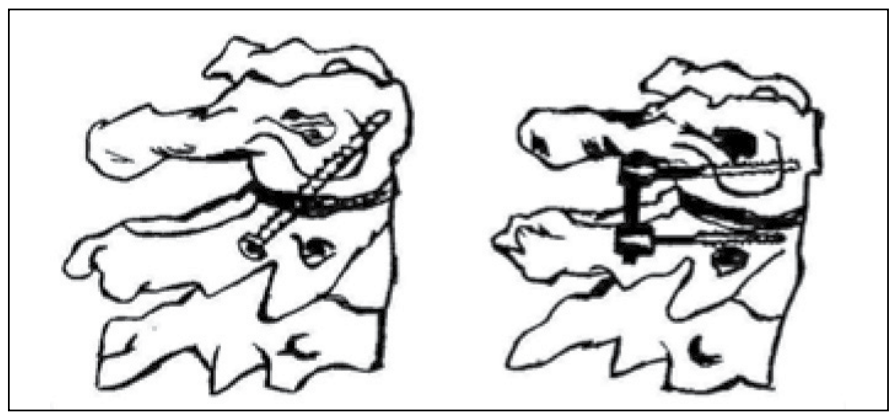

Figura 1. A esquerda técnica de Margerl e Seeman e a direita técnica de Harms e Melcher (desenho do autor).

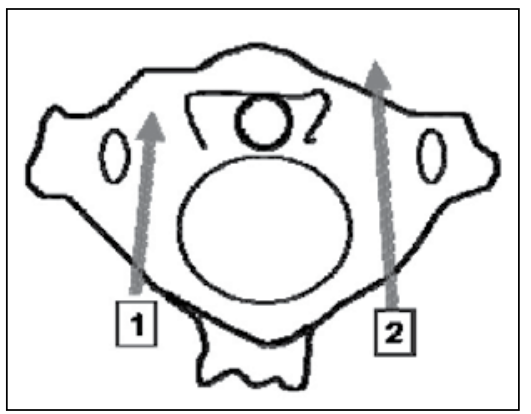

Figura 2. A seta 1 representa um parafuso uni cortical. A seta 2 representa um parafuso bi cortical.
Existem poucos estudos na literatura enfocando as relações entre a massa lateral de C1 e a artéria carótida interna e não há trabalhos que tenham empregado a técnica de Harms e Melcher ${ }^{5}$ com o objetivo de avaliar especificamente a trajetória dos parafusos e a sua relação com as artérias carótidas internas. O objetivo deste trabalho é estudar, em peças anatômicas; a relação entre os parafusos bicorticais pela técnica de Harms e Melcher ${ }^{5}$ e a artéria carótida interna, fornecendo informações úteis na avaliação da segurança dos métodos de fixação posterior antlantoaxial.

\section{MATERIAL E MÉTODOS}

Trata-se de um estudo do tipo experimental, descritivo, com uma amostra de conveniência composta por cinco segmentos cefálicos de cadáveres humanos formolizados (peças), fornecidos pelo Laboratório de Anatomia Neurocirúrgica da Faculdade de Medicina da Universidade Federal de Minas Gerais.

A dissecação posterior foi feita de acordo com a descrição de Campbell ${ }^{14}$. Em cada massa lateral de C1, direita e esquerda, foi inserido um parafuso, segundo a descrição original de Harms e Merchel $^{5}$, com o cuidado de exceder a cortical anterior da vértebra C1 em 3 a 7 mm, três passos da rosca. No total, dez parafusos foram colocados nas cinco peças anatômicas.

Realizou-se desarticulação da mandíbula e remoção em bloco de faringe e laringe, com preservação de todas as estruturas posteriores; a fascia buco faringea e a bainha carotídea.

A artéria carótida comum foi dissecada e em seguida feita canulação da artéria carótida interna, para uso do contraste no estudo tomográfico.

Para avaliação da posição exata do parafuso em cada massa lateral de $\mathrm{C} 1$, todas as peças foram submetidas a estudo tomográfico computadorizado multislice, com uso de contraste iodado na artéria carótida interna previamente canulada, utilizando equipamento da marca Philips, modelo Brilliance, com 64 canais. Foram realizadas aquisições volumétricas com reconstruções axiais de 1 $\mathrm{mm}$ de espessura.

Os músculos longos da cabeça, longos do pescoço, reto anterior da cabeça e reto lateral da cabeça foram dissecados para a mensuração das relações anatômicas anteriores (Figura 3).



Figura 3. Aspecto anterior das peças com exposição de parafusos sob a musculatura pré vertebral.

Posteriormente, os parafusos foram recuados para que a menor distância entre a borda medial da artéria carótida interna e o orifício de saída do parafuso fosse medida, em milímetros, utilizando compasso de alta precisão e paquímetro digital. Outra medida obtida foi a menor distancia entre borda posterior da artéria carótida interna 
e a cortical anterior ipsilateral do Atlas.

O objetivo de medir estas distâncias se dá pela importância da artéria carótida interna, que se lesionada torna-se o maior complicador do procedimento ${ }^{11}$.

\section{RESULTADOS}

As imagens de tomografia computadorizada analisadas mostraram que os dez parafusos se encontravam em posição adequada (Figuras 4 e 5)

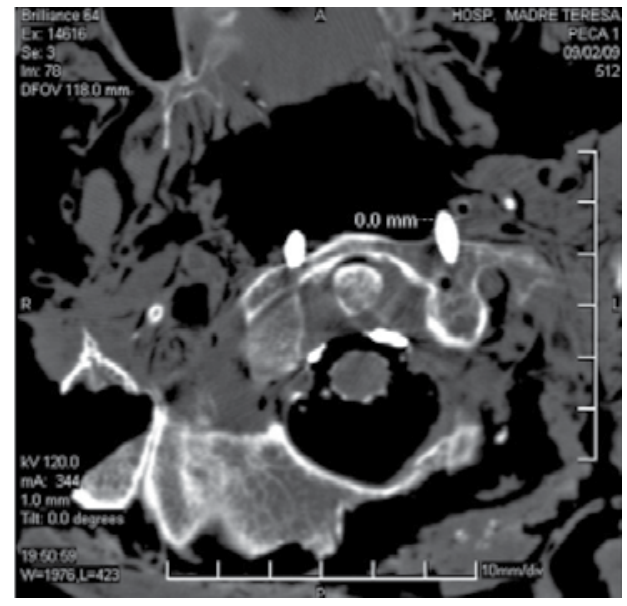

Figura 4. TC axial da peça 1 - note que o parafuso esquerdo toca a artéria carótida interna esquerda.

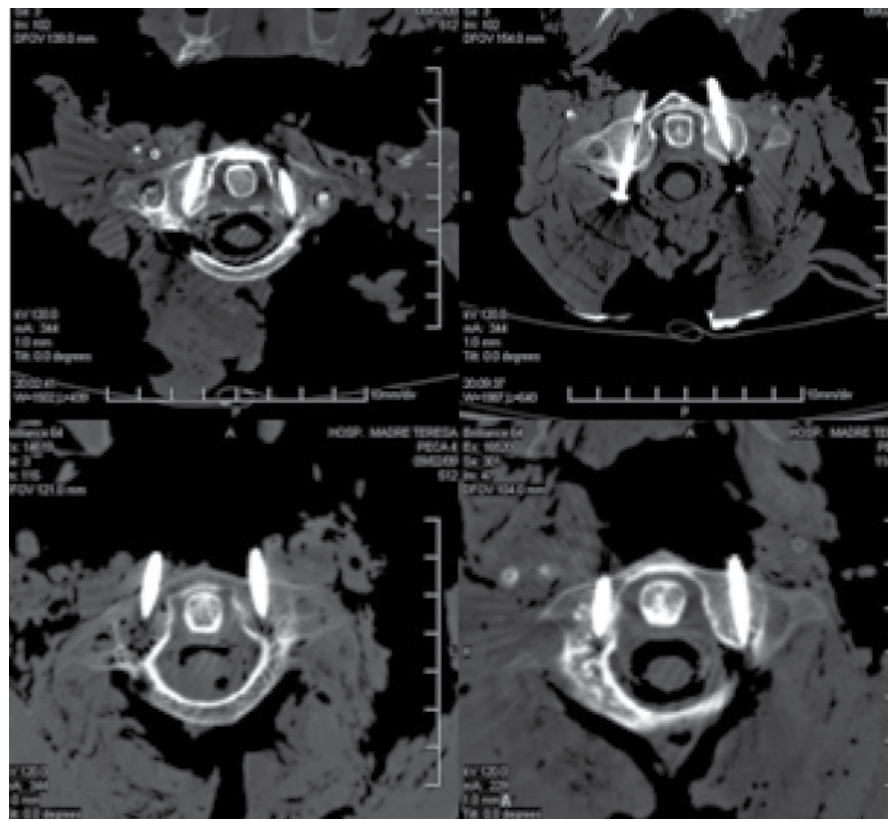

Figura 5. Cortes axiais da TC mostrando a posição correta dos parafusos em C1.

A média da menor distancia entre o orifício de saída do parafuso e a borda medial da artéria carótida interna direita foi de $11,55 \mathrm{~mm}$, com variação de 10,05 a 14,23 mm ), enquanto do lado esquerdo a média foi de 7,50 mm (variando de 2,75 a 12,42 mm)

A média da menor distancia entre a borda posterior da artéria carótida interna e a cortical anterior da massa lateral de $\mathrm{C} 1$ à direita foi de 4,24 mm (variando de 2,08 a 7,48 mm), enquanto do lado esquerdo a média obtida foi de 2,98 mm (com variação de 1,83 a $3,83 \mathrm{~mm}$ )
Os resultados obtidos se encontram na tabela abaixo (Tabela 1):

Tabela 1. Resultados das mensurações realizadas nos cadáveres

\begin{tabular}{c|c|c|c|c}
\hline \multirow{2}{*}{ Peça } & \multicolumn{2}{|c|}{ Distância $\mathbf{~ / ~ A C I ~}$} & \multicolumn{2}{c}{ Distância ACl /C1 } \\
\cline { 2 - 5 } & Direita & Esquerda & Direita & Esquerda \\
\hline 1 & $14,23 \mathrm{~mm}$ & $2,75 \mathrm{~mm}$ & $7,48 \mathrm{~mm}$ & $2,75 \mathrm{~mm}$ \\
\hline 2 & $11,53 \mathrm{~mm}$ & $12,42 \mathrm{~mm}$ & $4,27 \mathrm{~mm}$ & $3,83 \mathrm{~mm}$ \\
\hline 3 & $11,81 \mathrm{~mm}$ & $9,53 \mathrm{~mm}$ & $3,80 \mathrm{~mm}$ & $3,34 \mathrm{~mm}$ \\
\hline 4 & $10,05 \mathrm{~mm}$ & $8,60 \mathrm{~mm}$ & $3,55 \mathrm{~mm}$ & $3,20 \mathrm{~mm}$ \\
\hline 5 & $10,13 \mathrm{~mm}$ & $4,24 \mathrm{~mm}$ & $2,08 \mathrm{~mm}$ & $1,83 \mathrm{~mm}$ \\
\hline
\end{tabular}

$\mathrm{O}=$ orifício de saída do parafuso na cortical anterior de $\mathrm{C} 1$

$\mathrm{ACl}=$ artéria carótida interna $/ \mathrm{C} 1=$ cortical anterior de $\mathrm{C} 1$

Na análise tomográfica, observou-se que, na peça 1, o parafuso colocado na massa lateral esquerda tocou a artéria carótida interna (Figura 4), fato comprovado por visualização direta (Figura 6).
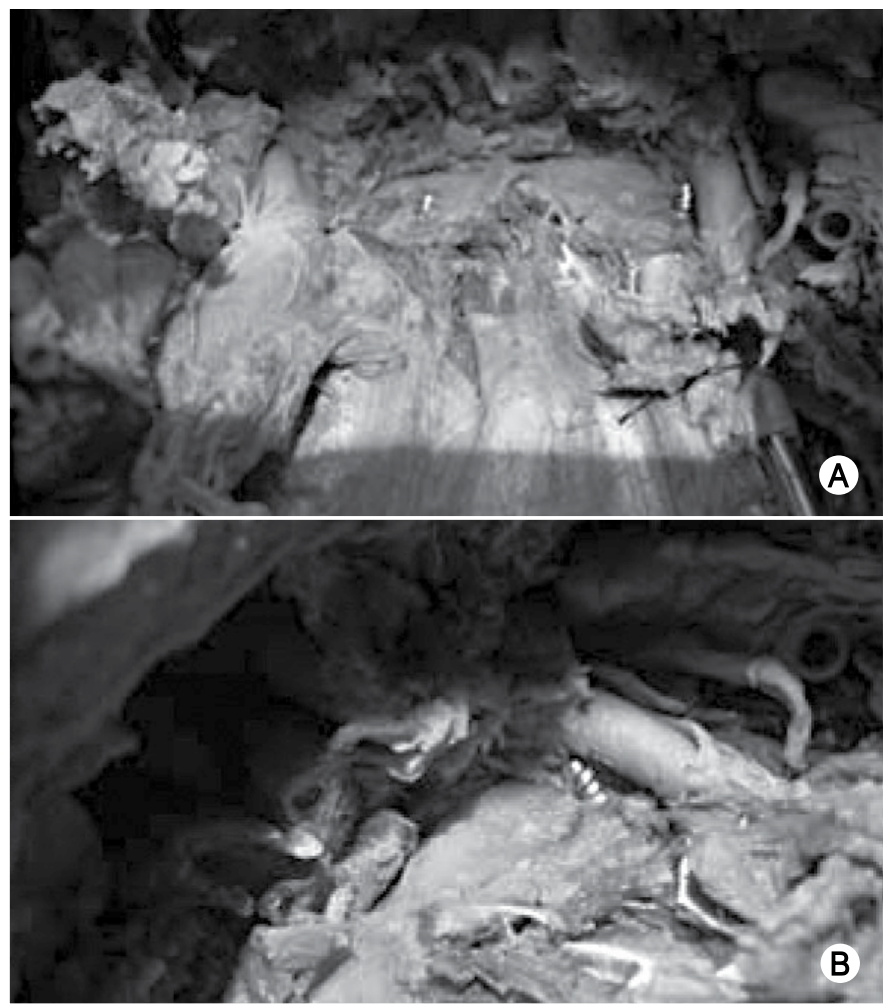

Figura 6. A - Peça 1- parafuso esquerdo tocando a ACl; B - foto em detalhe.

\section{DISCUSSÃO}

A artéria carótida interna se localiza sobre a lamina pré-vertebral da fascia, os músculos pré-vertebrais e sobre os processos transversos das vértebras cervicais superiores (da $1^{\mathrm{a}}$ à $3^{\mathrm{a}}$ vértebra cervical) ${ }^{21}$, inferindo que a artéria carótida interna encontra-se lateral à massa de $\mathrm{C} 1$.

Hoh et $\mathrm{al}^{22}$, em estudo tomográfico de cem pacientes, observaram que a $\mathrm{ACl}$ esquerda se encontrava na zona de risco, ou seja, na frente da massa lateral de $\mathrm{C} 1$, em 58 dos 100 pacientes, e a ACl direita em 74 dos 100 pacientes. A distancia entre a cortical anterior de $\mathrm{C} 1$ e a borda posterior da $\mathrm{ACl}$ no lado esquerdo foi de $3.5 \mathrm{~mm}$ $\pm 1.5(0.8-7 \mathrm{~mm})$ e à direita foi de $3.9 \pm 1.6 \mathrm{~mm}(1.2$ a $9.2 \mathrm{~mm})$.

Em 2003 Currier et al. ${ }^{12}$ relataram um caso de mau posiciona- 
mento do parafuso transarticular $\mathrm{C} 1$-C2 em relação à artéria carótida interna. Este relato motivou outros dois estudos realizados pelos mesmos autores ${ }^{12,23}$. As conclusões encontradas foram de que o ponto de saída do parafuso transarticular C1-C2 tem íntima relação com a artéria carótida interna, e, ao ser utilizada a técnica de parafusos bicorticais em $\mathrm{C} 1$ deve-se fazer de rotina uma tomografia contrastada para estudo da $\mathrm{ACl}$.

Em 2009, Simsek et al. ${ }^{24}$, em estudo tomográfico e anatômico, concluiu que a trajetória da artéria carótida interna é lateral ao ponto de entrada ideal do parafuso na massa lateral de C1 e que, quando aliada à angulação medial colocada no parafuso, a artéria carótida interna estaria relativamente segura. $\mathrm{Na}$ literatura ${ }^{25}$ e Carvalho et al. $^{26}$, em estudo anatômico encontrou resultados semelhantes, mostrando que a inclinação medial para a colocação do parafuso em C1 é necessária.

O estudo anatômico de Doherty e Heggeness ${ }^{27}$ apontou que o osso cortical mais denso do Atlas é encontrado anteriormente. Trabalho biomecânico de Eck et al. ${ }^{28}$ comparando o uso de parafusos bicortical versus unicortical na fixação da massa lateral de C1, mostrou diferença estatisticamente significativa, correspondente a $807 \mathrm{~N}$ versus $588 \mathrm{~N}$, respectivamente (P 0.008), contudo, ambos os valores são superiores aos valores necessários para a fixação da coluna cervical sub axial ${ }^{29}$.

$\mathrm{Na}$ avaliação dos autores ${ }^{30}$, ao se utilizar a técnica e Harms e Melcher ${ }^{5}$, as seguintes medidas tomográficas são úteis no planejamento pré operatório:

- Comprimento, largura, altura e angulação medial da massa lateral de C1.

- Relação da articulação atlanto axial e da cortical inferior do arco de C1.

- Posição e trajeto da artéria carótida interna ao nível da massa lateral de C1.

\section{REFERÊNCIAS}

1. Vender JR, Rekito AJ, Harrison SJ, McDonnell DE. The evolution of posterior cervical and occipitocervical fusion and instrumentation. Neurosurg Focus. 2004; 16(1):E9.

2. Smith MD, Kotzar G, Yoo J, Bohlman H. A biomechanical analysis of atlantoaxial stabilization methods using a bovine model. C1/C2 fixation analysis. Clin Orthop Relat Res. 1993;(290):285-95.

3. Dickman CA, Crawford NR, Paramore CG. Biomechanical characteristics of C1-2 cable fixations. J Neurosurg. 1996;85(2):316-22.

4. Hurlbert RJ, Crawford NR, Choi WG, Dickman CA. A biomechanical evaluation of occipitocervical instrumentation: screw compared with wire fixation. J Neurosurg. 1999;90(1 Suppl):84-90.

5. Harms J, Melcher RP. Posterior C1-C2 fusion with polyaxial screw and rod fixation. Spine (Phila Pa 1976). 2001:26(22):2467-71.

6. Magerl F, Seeman P. Stable posterior fusion of the atlas and axis by transarticular screw fixation. In: Kehr P,Weidner A, editors. The cervical spine. NewYork: Springer-Verlag; 1987. p. 322-7.

7. Coyne TJ, Fehlings MG, Wallace MC, Bernstein M, Tator $\mathrm{CH}$. C1-C2 posterior cervical fusion: long-term evaluation of results and efficacy. Neurosurgery. 1995; 37:688-93.

8. Brooks AL, Jenkins EB. Atlanto-axial arthrodesis by the wedge compression method. J Bone Joint Surg Am. 1978;60(3):279-84.

9. Dickman CA, Sonntag VK, Papadopoulos SM, Hadley MN. The interspinous method of posterior atlantoaxial arthrodesis. J Neurosurg. 1991;74(2):190-8.

10. Madawi AA, Casey AT, Solanki GA, Tuite G, Veres R, Crockard HA. Radiological and anatomic evaluation of the atlantoaxial transarticular screw fixation technique. J Neurosurg. 1997;86(6):961-8,

11. Farey ID, Nadkarni S, Smith N. Modified Gallie technique versus transarticular screw fixation in C1-C2 fusion. Clin Orthop Relat Res. 1999;(359):126-35.

12. Currier BL, Todd LT, Maus TP, Fisher DR, Yaszemski MJ. Anatomic relationship of the internal carotid artery to the $\mathrm{C} 1$ vertebra: a case report of cervical reconstruction for chordoma and pilot study to assess the risk of screw fixation of the atlas. Spine (Phila Pa 1976). 2003;28(22):E461-7.

13. Spetzler RF. Transoral approach to the upper cervical spine. In: Evarts CM, editor. Surgery of the musculoskeletal system. New York: Churchill Livingstone;1983.

14. Leventhal MR. Anatomia da coluna vertebral e vias de acesso cirúrgicas. In: Campbell Cirurgia ortopedica. Tradução de Tarcisio Eloy Pessoa de Barros Filho. Barueri, SP: Manole; 2006. p.1588-9.

15. Jun BY. Anatomic study for ideal and safe posterior C1-C2 transarticular screw fixation. Spine (Phila Pa 1976). 1998;23(15):1703-7.
- Dominância da artéria vertebral.

- Posição do forame transverso em C2.

- Comprimento, largura e altura do istmo do pedículo de C2 avaliados na provável trajetória dos parafusos $\left(20^{\circ}\right.$ a $30^{\circ}$ cefálico e medial).

No presente estudo, observou se que as distancias entre a cortical anterior de $\mathrm{C} 1 \mathrm{e}$ a borda posterior da $\mathrm{ACl}$ se assemelham às encontradas na literatura ${ }^{12,22,24}$. A medida da distância entre 0 orifício de saída do parafuso e a $\mathrm{ACl}$ é inédita considerando-se as principais bases de dados da literatura. Essas distâncias não apresentaram diferença estatística entre os lados direito e esquerdo. Na peça 1, à esquerda, o parafuso tocou a artéria carótida interna esquerda (10\% dos parafusos), tal fato se explicaria pela variação posicional da $\mathrm{ACl}$ em relação a massa lateral de $\mathrm{C}$ 1, estando esta em zona de risco ${ }^{17}$.

O pequeno tamanho amostral não permite inferências externas em relação aos resultados encontrados, entretanto, os enormes esforços logísticos impediram a obtenção de amostras maiores. Esta limitação é parcialmente compensada pela escassez de dados referentes a este tema na literatura e pelo aspecto inédito da utilização de parafusos na metodologia do estudo.

\section{CONCLUSÃO}

As imagens tomográficas realizadas no presente estudo permitiram uma avaliação adequada da relação entre os parafusos inseridos e a $\mathrm{ACl}$. Os resultados encontrados estão de acordo com os estudos similares existentes na literatura, que enfatizam a necessidade de uma avaliação imaginológica criteriosa da posição anatômica da artéria carótida interna, antes da utilização de parafusos bicorticais na massa lateral de C1 por via posterior.

16. Bogaerde MV, Viaene $P$, Thijs $V$. latrogenic perforation of the internal carotid artery by a transarticular screw: an unusual case of repetitive ischemic stroke. Clin Neurol Neurosurg. 2007;109(5):466-9.

17. Reilly TM, Sasso RC, Hall PV. Atlantoaxial stabilization: clinical comparison of posterior cervical wiring technique with transarticular screw fixation. J Spinal Disord Tech. 2003;16(3):248-53

18. Perren SM. The biomechanics and biology of internal fixation using plates and nails. Orthopedics.1989:12(1):21-33.

19. Schatzker J, Sanderson R, Murnaghan JP. The holding power of orthopaedic screws in vivo. Clin Orthop Relat Res. 1975;(108):115-6.

20. Uhthof HK. Current concepts of internal fixation of fractures. Can J Surg;1980: 23(3):214-6.

21. O'Rahilly R. O pescoço. In: Gardner G, Rahilly R. Anatomia; estudo regional do corpo humano. 4a. ed. Tradução de Rogerio Benevento. Rio de janeiro: Guanabara Koogan; 1988. p. 668-04.

22. Hoh DJ, Maya M, Jung A, Ponrartana S, Lauryssen CL. Anatomical relationship of the internal carotid artery to $\mathrm{C}-1$ : clinical implications for screw fixation of the atlas $\mathrm{J}$ Neurosurg Spine. 2008;8(4):335-40.

23. Currier BL, Maus TP, Eck JC, Larson DR, Yaszemski MJ. Relationship of the internal carotid artery to the anterior aspect of the C1 vertebra: implications for C1-C2 transarticular and C1 lateral mass fixation. Spine (Phila Pa 1976). 2008;33(6):635-9.

24. Simsek S, Yigitkanli K, Turba UC, Comert A, Seçkin H, Tekdemir I, Elhan A. Safe zone for $\mathrm{C} 1$ lateral mass screws: anatomic and radiological study. Neurosurgery. 2009;65(6):1154-60.

25. Wang MY, Samudrala S. Cadaveric morphometric analysis for atlantal lateral mass screw placement. Neurosurgery. 2004;54(6):1436-9;

26. Carvalho MF, Rocha RT, Monteiro JT., Pereira CU, Leite RF, Defino HL. Estudo tomográfico do atlas relacionado com a inserção de implantes na massa lateral Acta Ortop Bras. 2009;17(3):136-8.

27. Doherty BJ, Heggeness MH. The quantitative anatomy of the atlas. Spine (Phila Pa 1976). 1994;19(22):2497-500.

28. Eck JC, Walker MP, Currier BL, Chen Q, Yaszemski MJ, An KN.. Biomechanical comparison of unicortical versus bicortical $\mathrm{C} 1$ lateral mass screw fixation. J Spinal Disord Tech. 2007;20(7):505-8

29. Heller JG, Estes BT, Zaouali M, Diop A. Biomechanical study of screws in the lateral masses: variables affecting pull-out resistance. J Bone Joint Surg Am. 1996;78(9):1315-21.

30. Abreu EV, Simões MS, Brasil AV, Sanchis FG. Fixação posterior C1-C2 com sistema de parafuso e haste - experiência em cinco casos. Coluna/Columna. 2007;6(1):12-7. 